\title{
ATYPICAL HEMOLYTIC UREMIC SYNDROME WITH OBSTRUCTIVE HYDROCEPHALUS IN AN INFANT
}

Sahajananda H${ }^{1}$, Kayalvizhi K. B², K. T. Venkateshmurthy33, Karthik G. S4, Sudheer R5

\section{HOW TO CITE THIS ARTICLE:}

Sahajananda H, Kayalvizhi K. B, K. T. Venkateshmurthy, Karthik G. S, Sudheer R. "Atypical Hemolytic Uremic Syndrome with Obstructive Hydrocephalus in an Infant". Journal of Evolution of Medical and Dental Sciences 2015; Vol. 4, Issue 41, May 21; Page: 7249-7254, DOI: 10.14260/jemds/2015/1052

ABSTRACT: Hemolytic uremic syndrome (HUS) which is characterized by a triad of hemolytic anemia, low platelet count and acute renal failure was first described by Gasser and others in $1955 .(1,2)$ Initially it used to have a mortality rate of $50 \%$ to $80 \%$. But the improved diagnostic methods and treatment strategies has reduced the mortality to $2 \%$ - $8 \%$. Three types of HUS have been identified, typical HUS after enterohemorrhagic. Escherichia coli, shiga toxin 1 \& 2 (Stx-HUS/D+HUS) producing bloody diarrhea, atypical HUS (a HUS/D-HUS) with complement abnormalities and not associated with diarrhea and pneumococcal associated HUS-mediated by neuraminidase in the presence of streptococcus pneumonia infection. Multiple systems are involved in the pathology including gastrointestinal, renal, hematological, brain, pancreas and myocardium. ${ }^{(3,4,5)}$ Here we present a case of an infant with atypical HUS who also developed obstructive hydrocephalus following respiratory infection due to streptococcus pneumonia. This is the first case report in the English literature so far.

KEYWORDS: Atypical HUS, Paediatric, Obstructive Hydrocephalus.

CASE REPORT: An eight month old female child weighing 8kgs presenting with h/o fever, cough, difficulty in breathing for 3 days, 2 episodes of convulsions and anuria was admitted to paediatric ICU. On examination child was moderately built and nourished with normal developmental milestones. It was drowsy with sluggishly reacting and irregular pupils. Room air saturation was $86 \%$. Child had acute renal failure and metabolic acidosis. Child was intubated with size $4 \mathrm{~mm}$ Endotracheal tube and electively ventilated (Fig). Blood investigations revealed Anemia with $\mathrm{Hb}$ of $5.7 \mathrm{gm} / \mathrm{dl}$, PCV of 13.2, WBC count of 4000/cumm., Platelets were 17,000/cumm, Baby had raised PT and APTT with INR of 1.76. Peripheral smear showed fragmented RBCs like schistocytes and schizocytes. Blood urea was 104 , creatinine was 1.2 , Total bilirubin was 1.5 , direct bilirubin was 1.3 , AST was 159, ALT was 40, Total proteins were 5.7 with serum Albumin of 2.8. Blood culture was positive for $\alpha$ hemolytic streptococci. Ophthalmoscopic examination revealed venous congestion of both the eyes.

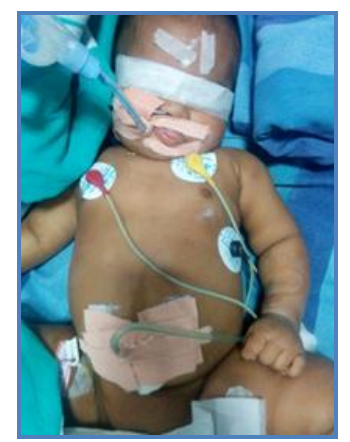

\section{Fig. 1: Intubated child on the first day}


Baby was diagnosed to be suffering from atypical HUS and started on Inj. Meropenam150 mg twice a day (BID), Inj. Phenytoin $30 \mathrm{mg}$ i.v BID, $1.5 \mathrm{ml}$ of Domstal syrup was administered through the Ryle's tube thrice a day. Budecort nebulization was administered four times a day (QID).

Baby was transfused 2 units of platelets on second day and 1 unit on third day.70 ml of packed cells and $120 \mathrm{ml}$ of Fresh frozen plasma was transfused on second and third day. Baby was treated with antihypertensives to control the hypertension using Tab. Nifedepine 4mg QID, Methyldopa $20 \mathrm{mg}$ QID and Hydralazine $22.5 \mathrm{mg}$ TID. Peritoneal dialysis was carried out for 14 days to treat the renal injury.

Serological tests revealed C3 1.2gm/dl, C5 0.8gm/dl, Factor B $250 \mu \mathrm{g} / \mathrm{ml}$, Factor H 1.5mg/dl. Magnetic resonance imaging (MRI) brain on day 4 showed multiple bilateral infarcts in cerebral hemispheres, caudate nuclei, thalamus, lentiform nuclei with dilatation of supratentorial ventricles and communicating hydrocephalus. Inj. Dexamethasone $1.5 \mathrm{mg}$ was administered BID and Hypertonic saline infusion was instituted to control the suspected ischemic insult.

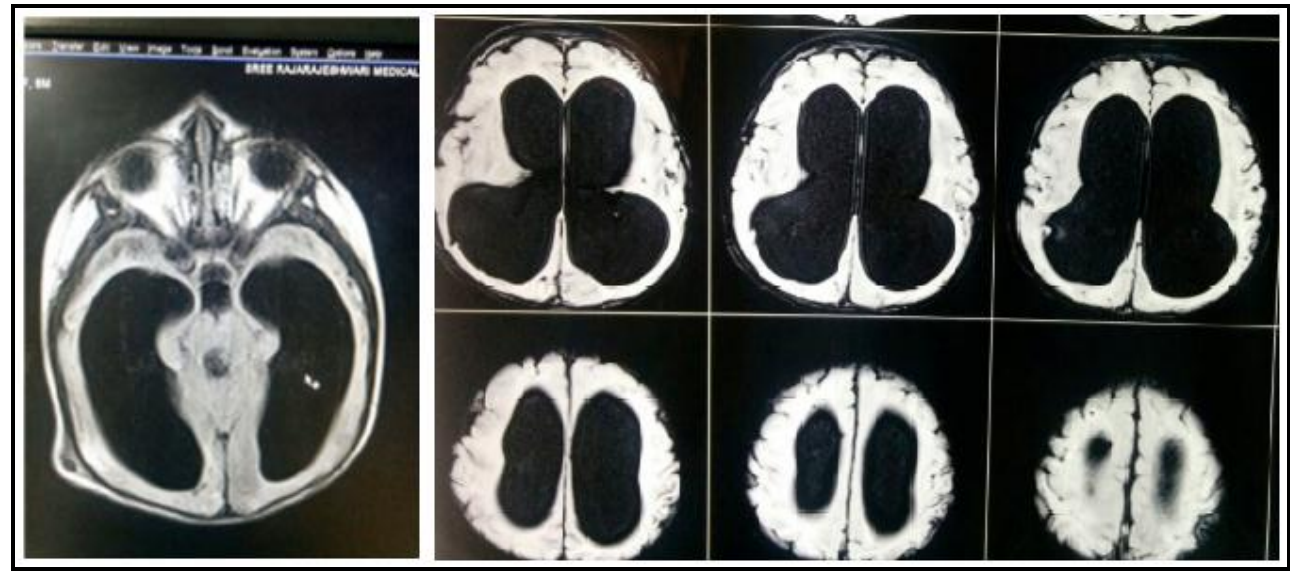

Fig. 2: This is the MRI film image with enlarged ventricles and caudate nuclei

Since the cranial size was gradually increasing with bulging fontanelle, MRI was repeated on $15^{\text {th }}$ day, which showed obstructive hydrocephalus. The Neurosurgeon planned ventriculo Peritoneal shunt and baby was shifted to Operation, after taking informed consent from the parents. After medical optimization, her blood investigations prior to surgery were: $\mathrm{Hb} 9.3 \mathrm{gm} / \mathrm{dl}$, platelets 1.3 lakhs, urea $10 \mathrm{mg} / \mathrm{dl}$, creatinine $0.2 \mathrm{mg} / \mathrm{dl}$, Serum sodium 132, Potassium 3.9, Serum Chloride 99. Baseline parameters in the Operation theatre were: Pulse rate $140 / \mathrm{min}$, Blood Pressure96/58mmH, and oxygen saturation was $98 \%$.

Baby was premedicated with inj. Glycopyrolate $0.05 \mathrm{mg}$ i.v, Midazolam 0.2mg i.v and Fentanyl $10 \mu \mathrm{g}$ i.v. General anaesthesia was induced with $40 \mathrm{mg}$ of inj. Thiopentone i.v and maintained with inj. Atracurium $4 \mathrm{mg}$ i.v along with Sevoflurane 1.5\% in Oxygen. Pediatric Fibre optic bronchoscopy was performed to rule out glottis stenosis. Endotracheal tube was changed and reintubated with new $4 \mathrm{~mm}$ size endotracheal tube. Baby warmer was used to maintain body temperature. During tunneling phase of the surgery, anesthetic depth was increased with sevoflurane. All the vital parameters were stable throughout the procedure. Baby was shifted back to Pediatric intensive care unit after Ventriculo Peritoneal shunt surgery for further management. CSF analysis showed normal values and no cells. 
On $24^{\text {th }}$ day of admission baby was weaned off the ventilator and extubated. 2 days later baby was reintubated because of difficulty in breathing. Tracheostomy was performed on $31^{\text {st }}$ day. As the child showed gradual improvement and became haemodynamically stable, all the medications were tapered from $38^{\text {th }}$ onwards. Tracheostomy tube was removed and wound closed on 40th day. The child was discharged on $50^{\text {th }}$ day after it became physically active with social smile, suckling, handgrip, sitting with support, normal head size.

One month later, baby came for a follow up at which time the baby was normal in behavior with no neurological deficits. Ventriculo-Peritoneal shunt was functioning well and head size was normal. Further follow up was advised once in 3 months or on relapse of symptoms, whichever is earlier. This is the picture of the child during follow up.

Fig. 3: Child with tracheostomy tube on $31^{\text {st }}$ day.

Fig. 4: After removal of tracheostomy tube on $41^{\text {st }}$ day.

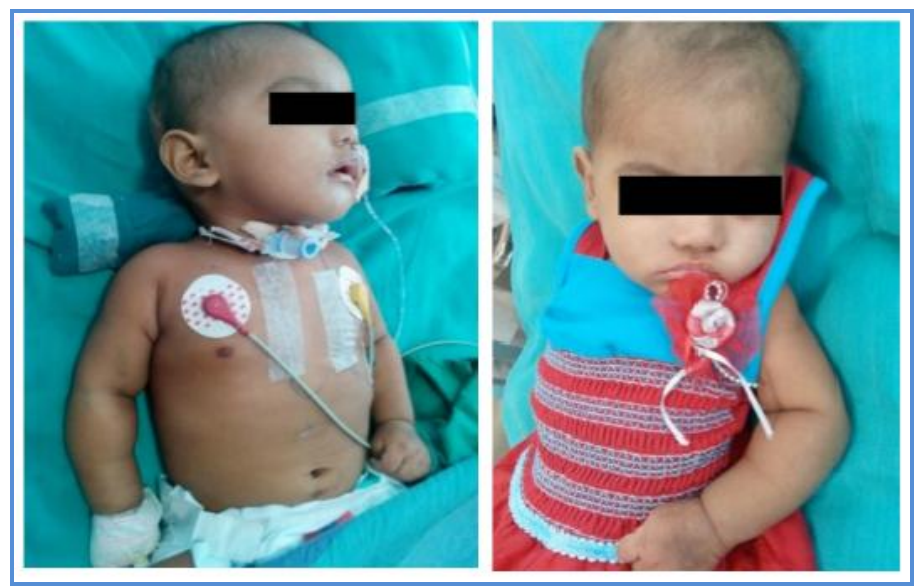

Fig. 3 \& 4

DISCUSSION: Hemolytic uremic syndrome (HUS) is defined by the triad of micro angiopathic hemolytic anemia, thrombocytopenia and renal impairment. Atypical HUS (aHUS) defines non Shigatoxin-HUS and even if some authors include secondary aHUS due to Streptococcus pneumoniae or other causes, aHUS designates a primary disease due to a disorder in complement alternative pathway regulation. Atypical HUS represents $5-10 \%$ of HUS in children, but the majority of HUS are in adults. The incidence of complement-aHUS is not known precisely. However, more than 1000 aHUS patients investigated for complement abnormalities have been reported. Onset is from the neonatal period to the adult age. Most patients present with hemolytic anemia, thrombocytopenia and renal failure and $20 \%$ have extra renal manifestations.

Two to $10 \%$ die and one third progress to end-stage renal failure at first episode. Half of patients have relapses. Mutations in the genes encoding complement regulatory proteins factor $\mathrm{H}$, membrane cofactor protein (MCP), factor I or thrombomodulin have been demonstrated in 20-30\%, $5-15 \%, 4-10 \%$ and $3-5 \%$ of patients respectively, and mutations in the genes of C3 convertase proteins, C3 and factor $\mathrm{B}$, in $2-10 \%$ and $1-4 \%$. In addition, $6-10 \%$ of patients have anti-factor $\mathrm{H}$ antibodies. Diagnosis of aHUS relies on 1) No associated disease 2) No criteria for Shigatoxin-HUS (Stool culture and PCR for Shiga-toxins; serology for anti-lipopolysaccharides antibodies) 3) No criteria for thrombotic thrombocytopenic purpura (Serum ADAMTS 13 activity>10\%). 
Investigation of the complement system is required $(\mathrm{C} 3, \mathrm{C} 4$, factor $\mathrm{H}$ and factor I plasma concentration, MCP expression on leukocytes and anti-factor $\mathrm{H}$ antibodies; genetic screening to identify risk factors). The disease is familial in approximately $20 \%$ of pedigrees, with an autosomal recessive or dominant mode of transmission. As penetrance of the disease is $50 \%$, genetic counseling is difficult. Plasmatherapy has been first line treatment until presently, without unquestionable demonstration of efficiency. There is a high risk of post-transplant recurrence, except in MCP-HUS. Case reports and two phase II trials show an impressive efficacy of the complement C5 blocker eculizumab, suggesting it will be the next standard of care. Except for patients treated by intensive plasmatherapy or eculizumab, the worst prognosis is in factor H-HUS, as mortality can reach $20 \%$ and $50 \%$ of survivors do not recover renal function. Half of factor I-HUS progress to end-stage renal failure. Conversely, most patients with MCP-HUS have preserved renal function. Anti-factor $\mathrm{H}$ antibodies-HUS has favourable outcome if treated early. ${ }^{1}$

HUS which involves many systems in the body with variants like Stx-HUS, atypical HUS, Thrombotic thrombocytopenic purpura, and neuraminidase associated HUS. Though most of the cases of HUS follows Escherichia coli, 0-157:H7 enteritis, other shiga toxin producing Ecoli also have been identified. Though the disease complex is known to occur in children above 5 years of age up to $30 \mathrm{yrs}$, atypical HUS has been diagnosed in children of 6 months also with high mortality rate. ${ }^{5}$

Atypical HUS is more known to cause mutations in complement system and dysregulation. $(5,6)$ Central nervous system involvement affects 20\%- 50\% of patients with increased morbidity and mortality. $(7,8)$ Recent advances in diagnosing and managing strategies like treating HUS with plasma therapy, platelets, hemo/peritoneal dialysis, shiga toxin antibodies, anti C5 monoclonal antibodies (Eculizumab), anti-epileptic levetiracetam has dramatically reduced the mortality rate to $<10 \% .(9,7,8)$ Toxin mediated cerebral vasculopathy and thrombotic micro angiopathy have been found to cause hypoxic encephalopathy along with anuric acute renal failure.(10)

This patient presented with severe respiratory tract infection with $\alpha$ hemolytic streptococci followed by anuria and convulsions. MRI Brain revealed hypoxic injury which later led to obstructive hydrocephalus. Many complications with CNS involvement in HUS have been reported earlier including hemorrhagic and non hemorrhagic infarctions, cerebral edema, gliosis,(11) but no case has been reported in the literature with obstructive hydrocephalus.

In a review of the clinical and neurophysiological features of CNS involvement during HUS in 22 children seizures, alone or as part of neurological involvement, were associated with mortality or long term neurological sequelae.(12)

Aggressive medical management along with timely surgical intervention helped in our case. Delay in diagnosing the thrombotic microangiopathy in the brain due to neuraminidase aHUS might have caused the obstruction for the absorption of CSF leading to hydrocephalus in our case.

This patient had obstruction in the absorptive pathway of Cerebrospinal fluid in the ventricles which gradually presented with dilated ventricles and caudate nuclei (Fig. 2), which was successfully treated with Ventriculo peritoneal shunt following higher antibiotic, platelet transfusion, peritoneal dialysis, FFP s and packed cells along with a steroid.

CONCLUSION: HUS should be suspected in children who present with h/o fever, watery diarrhea, followed by bloody diarrhea, vomiting, anuria, increasing hypertension, convulsions, loss of consciousness. Severe streptococcal pneumonia producing neuraminidase also has been found to cause atypical HUS. 


\section{CASE REPORT}

Brain MRI is an essential tool to differentiate neurological complications due to thrombotic microangiopathy and CNS complications of hypertension. (Reversible Posterior leucoencephalopathy syndrome-RPLS) and guide therapeutic strategy. Peritoneal dialysis, blood pressure control, intensive therapy with plasma exchange, platelets, CSF draining with VP shunt successfully reverses all the complications of atypical HUS.

\section{REFERENCES:}

1. Loirat C, Frémeaux-Bacchi V. Atypical hemolytic uremic syndrome. Orphanet J Rare Dis 2011: 60.

2. Gasser, C., et al. "Hemolytic-uremic syndrome: bilateral necrosis of the renal cortex in acute acquired hemolytic anemia." Schweizerische medizinische Wochenschrift 85. 38-39. (1955): 905.

3. Corrigan, James J., and Frank G. Boineau. "Hemolytic-uremic syndrome. Paediatrics in review 22.11(2001): 365- 369.

4. Karmalli M A, Petrick $M$ et al. The association between hemolytic uremic syndrome and infection by verotoxin-producing Escherichia coli. J Infect Dis 1985; 151: 775-82.

5. Naris M Mescia F, Remuzzi G Stec HUS, atypical HUS and TTP are all diseases of complement activation Nat Rev Nephro 2012: 8: 622-633.

6. De Jorge EG,Hill A et all.Development of atypical HUS depends on complement C5.J Am soc Nephrol 2011.22:137-145.

7. Salvadori, Maurizio, and Elisabetta Bertoni. "Update on hemolytic uremic syndrome: diagnostic and therapeutic recommendations." World journal of nephrology 2.3 (2013): 56.

8. Koehl, Bérengère, et al. "Neurological involvement in a child with atypical hemolytic uremic syndrome." PEriksson KJ, Boyd SG, Tasker RC. 2001. Acute neurology and neurophysiology of haemolytic-uraemic syndrome. Arch. Dis. Child. 84:434-435.ediatric nephrology 25.12 (2010): 2539-2542.

9. Schering J, Andreoli SP, Zimmerhackl LB. Treatment and outcome jf Shiga-toxin- associated hemolytic uremic syndrome (HUS). Pediatr. Nefrol. 2008; 23: 1749-1760.

10. Signorini E1, Lucchi S, Mastrangelo M, Rapuzzi S, Edefonti A, Fossali E. Central nervous system involvement in a child with hemolytic uremic syndrome Pediatr Nephrol. 2000 Sep: 14(10-11): 990-2.

11. Theobald I1, Kuwertz-Bröking E, Schiborr M, Heindel W. Central nervous system involvement in hemolytic uremic syndrome (HUS)-a retrospective analysis of cerebral CT and MRI studies. Clin Nephrol. 2001 Dec: 56(6): S3-8.

12. Eriksson KJ, Boyd SG, Tasker RC. 2001. Acute neurology and neurophysiology of haemolyticuraemic syndrome. Arch. Dis. Child. 84: 434-435. 


\section{AUTHORS:}

1. Sahajananda $\mathrm{H}$.

2. Kayalvizhi K. B.

3. K. T. Venkateshmurthy

4. Karthik G. S.

5. Sudheer R.

\section{PARTICULARS OF CONTRIBUTORS:}

1. Professor \& HOD, Department of Anaesthesia, Rajarajeswari Medical College \& Hospital, Bangalore.

2. Assistant Professor, Department of Anaesthesia, Rajarajeswari Medical College \& Hospital, Bangalore.

3. Professor, Department of Anaesthesia, Rajarajeswari Medical College \& Hospital, Bangalore.

FINANCIAL OR OTHER COMPETING INTERESTS: None
4. Associate Professor, Department of Anaesthesia, Rajarajeswari Medical College \& Hospital, Bangalore.

5. Assistant Professor, Department of Anaesthesia, Rajarajeswari Medical College \& Hospital, Bangalore.

\section{NAME ADDRESS EMAIL ID OF THE CORRESPONDING AUTHOR:}

Sahajananda $\mathrm{H}$,

\# 413, Ragam, $7^{\text {th }}$ Main,

Vijaya Bank Colony, Bannerghatta,

Bangalore-76.

E-mail: sahaj_anand@hotmail.com

Date of Submission: 12/03/2015.

Date of Peer Review: 13/03/2015.

Date of Acceptance: 13/05/2015.

Date of Publishing: 21/05/2015. 